Revista Destaques Acadêmicos, Lajeado, v. 10, n. 3, 2018. ISSN 2176-3070

DOI: http://dx.doi.org/10.22410/issn.2176-3070.v10i3a2018.1887

http://www.univates.br/revistas

\title{
ASSOCIAÇÃO ENTRE EQUILÍBRIO E CAPACIDADE FUNCIONAL EM MULHERES IDOSAS
}

\author{
Rômulo Augusto Scherer ${ }^{1}$, Jéssica Luana Dornelles da Costa ${ }^{2}$, \\ Fabian Arruda Barbosa ${ }^{3}$, Bruno De Maman ${ }^{4}$, Caroline Pieta Dias ${ }^{5}$, \\ Carlos Leandro Tiggemann ${ }^{6}$
}

Resumo: A população brasileira vem envelhecendo de forma rápida, sendo os idosos o estrato da população mais sedentária da sociedade, o que pode levar a um declínio na qualidade de vida. $\mathrm{O}$ objetivo deste estudo foi verificar a associação de equilíbrio e capacidade funcional em mulheres idosas. Método: Trata-se de estudo descritivo correlacional, constituído por uma amostra de 31 idosas, com idades entre 60 e 75 anos, todas saudáveis. Foram realizados dois testes para avaliar o equilíbrio: Escala de Equilíbrio de Berg - EEB e Baropodometria (em quatro posições); três para capacidade funcional: Timed Up-and-go - TUG; teste de subir degraus; teste de sentar e levantar em uma cadeira. A análise estatística foi realizada por meio da estatística descritiva (média \pm desvio padrão) e correlação de Pearson (SPSS, $\mathrm{p} \leq 0,05$ ). Resultados: Somente foi encontrada uma alta, negativa e significativa correlação entre TUG e EEB $(\mathrm{r}=-0,710 ; \mathrm{p}<0,01)$, indicando que quanto menor o tempo de execução no teste TUG, maior a pontuação no teste de equilíbrio de EEB. As demais variáveis não apresentaram correlação significativa. Conclusão: Conclui-se com o presente estudo que somente o teste TUG apresentou associação com o teste de EEB, indicando que o equilíbrio possa ter importante função nesta capacidade funcional.

Palavras-chave: Idosas, equilíbrio, capacidade funcional.

1 Graduado em Educação Física Bacharelado - Universidade do Vale do Taquari-Univates.

2 Graduada em Educação Física Licenciatura e Bacharelado -Universidade do Vale do TaquariUnivates.

3 Graduado em Educação Física Bacharelado - Centro Universitário Univates.

4 Graduado em Educação Física Bacharelado - Universidade do Vale do Taquari-Univates

5 Doutora em Ciência do Movimento Humano - Universidade Federal do Rio Grande do Sul (UFRGS).

6 Doutor em Ciência do Movimento Humano - Universidade Federal do Rio Grande do Sul (UFRGS). 


\section{INTRODUÇÃO}

O aumento da população idosa tem aumentado de forma acelerada, de acordo com os dados do Ministério da Saúde (2007), estimando-se 32 milhões de pessoas idosas para 2025. Com o envelhecimento acontecem perdas substanciais de funções, podendo acarretar o surgimento de doenças crônicas e até a morte (PATERSON; WARBURTON, 2010). Além disso, sabe-se que a qualidade de vida pode se associar a outros fatores como moradia, saúde, capacidade funcional, saneamento básico, alimentação, transporte, educação, lazer, tabagismo, stress, condições de moradia inadequada, sedentarismo e obesidade (BOUCHARD, 2003).

Esta população idosa está entre os sedentários e inativos da sociedade, o que pode levar a um declínio na qualidade de vida e a uma dependência funcional (PATERSON; WARBURTON, 2010). Incapacidade funcional pode ser definida como a dificuldade de realizar atividades típicas e pessoalmente desejada, sendo que a avaliação da funcionalidade consiste no desempenho de atividades e funções em diferentes áreas, dentre as quais as tarefas da vida cotidiana, as interações sociais, as atividades de lazer e outras requisições do dia-a-dia (ARAUJO et al., 2010).

Do ponto de vista funcional, a população de indivíduos idosos caracteriza-se, entre outros aspectos, por um decréscimo do sistema neuromuscular, verificando-se a perda de massa muscular, debilidade do sistema muscular, redução da flexibilidade, da força, da resistência e da mobilidade articular, fatores que, por decorrência, determinam limitação da capacidade de coordenação e de controle do equilíbrio corporal estático e dinâmico (REBELLATO et al., 2005). Os sistemas responsáveis pelo equilíbrio se alteram com o envelhecimento, sendo que cerca de $65 \%$ dos idosos têm alguma sensação de perda de equilíbrio (ARAUJO et al., 2010). O equilíbrio é um processo complexo, dependente da combinação da sensação da visão, dos comandos centrais e das respostas neuromusculares e, especificamente, da forca muscular e proprioceptores. Para os idosos manterem o equilíbrio, há a necessidade de atenção, memória, audição, propriocepção, visão e controle postural, habilidades estas que tendem a reduzir com o envelhecimento (NETO et al., 2015).

Em relação à associação entre equilíbrio e capacidade funcional em idosas, a literatura nos traz alguns estudos. O estudo de Souza et al. (2011) mostra que há correlação entre equilíbrio e capacidade funcional em idosas com vestibulopatias periféricas, e além disto, que uma pior capacidade funcional representa um maior riscos de queda. Já o estudo de Gonçalves et al. (2009) aponta que idosos com histórico de quedas demostraram comprometimento na avaliação do equilíbrio funcional em relação às idosas sem histórico de quedas, supondo que o histórico de quedas seja um fator de incapacidade funcional. Ainda, o estudo de Ribeiro et al. (2009) referiu que idosos ativos foram melhores 
tanto nos testes de equilíbrio como nos testes de capacidade funcional havendo correlação entre os mesmos.

Em contrapartida aos estudos supracitados, o estudo de Silva et al. (2014) concluiu que idosos sem histórico de quedas não tiveram um melhor desempenho nos testes, quando comparados aos idosos com histórico de quedas. Os mesmos resultados foram encontrados no estudo de Rodrigues (2015) que apontou não haver diferença entre capacidade funcional e equilíbrio em idosas com histórico de quedas no último ano e idosas sem histórico de quedas. Assim, visto que a literatura traz algumas controvérsias sobre o assunto, este estudo tem como objetivo, verificar a relação entre o equilíbrio e a capacidade funcional em mulheres idosas.

\section{MÉTODO}

A pesquisa classifica-se como descritiva correlacional. Os participantes assinaram um Termo de Consentimento Livre Esclarecido (TCLE), no qual estavam cientes do estudo e concordavam em participar da pesquisa de livre e espontânea vontade. Este estudo foi aprovado pelo Comitê de Ética e Pesquisa (COEP) da Universidade do Vale do Taquari - Univates (parecer número 1.567.120) Todos os procedimentos foram aplicados no Laboratório de Fisiologia do Exercício da Universidade do Vale do Taquari - Univates,

O estudo foi composto por 31 mulheres com idades entre 60 e 78 anos, recrutadas via meios de comunicação como redes sociais, panfletos e visita a centros de convivência de idosos. Foram adotados como critérios de inclusão para participação do estudo: (1) ser saudável mediante o preenchimento negativo do questionário de prontidão para atividade física; e (2) não estar em tratamento à base de medicamentos de uso contínuo ou eventual que afetasse o equilíbrio. O nível de atividade física foi avaliado por meio do Questionário Internacional de Atividade Física (IPAQ, 2005) e as medidas corporais mediante avaliação padronizada do laboratório. Desta forma, a amostra foi constituída por mulheres com idade média de $66,65 \pm 5,13$ anos, a massa corporal foi de $69,52 \pm 12,91 \mathrm{~kg}$, a estatura de 1,54 $\pm 0,06$ metros e o índice de massa corporal (IMC) de 29,19 $\pm 5,29 \mathrm{~kg} / \mathrm{m}^{2}$. Na classificação do IMC, 25,8\% das idosas foram classificadas como peso normal, $22,6 \%$ com sobrepeso e $51,6 \%$ como obesas. Quanto ao nível de atividade física, 74,2\% foram consideradas ativas e $25,8 \%$ sedentárias.

Os testes de capacidade funcional e equilíbrio foram aplicados em dois dias diferentes no turno da tarde, sendo os mesmos replicados (retestes) uma semana após. Tal estratégia foi adotada visando minimizar a influência do fator familiarização aos testes, sendo utilizados apenas os dados dos retestes para a análise. A avaliação da capacidade funcional consistiu de três testes físicos, baseados em atividades motoras realizadas no dia a dia de idosos, sendo eles: teste de subir degraus (DEG), teste de sentar e levantar em uma cadeira (CAD) 
e o Timed Up and Go (TUG). Todos os testes foram aplicados na velocidade máxima que cada idosa conseguisse realizar de forma segura e sem auxílio. Duas tentativas foram realizadas, com intervalo de 3 minutos entre cada uma, sendo registrado o menor tempo (TIGGEMANN et al., 2016).

No teste DEG, as idosas subiram um lance de 10 degraus sem o uso do corrimão. Cada degrau 0,18 m de altura e 0,26 m de comprimento, e os sujeitos pisaram em um degrau a cada vez. O tempo foi registrado a partir do primeiro contato feito no primeiro degrau e finalizado ao ocorrer o contato com o último degrau. No teste $\mathrm{CAD}$, as idosas levantaram e sentaram em uma cadeira (assento plano com $43 \mathrm{~cm}$ altura) durante 5 repetições, sendo o tempo registrado ao comando de iniciar o primeiro levantamento (com o sujeito iniciando sentado) e no final (ao sentar após o $5^{\circ}$ levantamento). As idosas deviam permanecer com os braços cruzados em frente ao tórax, realizar a extensão completa de joelhos, quadris e coluna ao levantar, e ao sentar apoiar completamente as costas no encosto da cadeira. Para o teste TUG, o mesmo iniciou a partir da posição sentada em uma cadeira ( $43 \mathrm{~cm}$ de altura, com costas apoiadas) as participantes) levantaram da mesma (sem auxílio das mãos), caminhar e contornar um cone posicionado no solo a $3 \mathrm{~m}$ de distância e voltar a sentar-se (com as costas apoiadas), sendo cronometrado e registrado o menos tempo.

A avaliação de equilíbrio consistiu em dois testes, sendo eles a Baropodometria (Baro) e a Escala de Equilíbrio de Berg (EEB). O teste da EEB serviu para avaliar o equilíbrio funcional, com base em 14 itens comuns para a vida cotidiana. A pontuação máxima a ser alcançado nesta escala é 56 e cada item tem uma escala ordinal que consiste em cinco opções variando de 0 a 4 pontos de acordo com o nível de dificuldade (quanto maior a pontuação, melhor a equilibrar). O teste é simples, fácil de administrar, e seguro para a avaliação de sujeitos idosos (BERG, et al., 1989).

O teste da Baro foi realizado através do baropodômetro eletrônico (Footwork IST Informatique da marca Arkipélago. Consiste em uma base rígida com dimensões de $575 \times 450 \times 25 \mathrm{~mm}$, constituído de 2.704 sensores capacitivos piezelétricos de pressão de $7,62 \times 7,62 \mathrm{~mm}$, capazes de registrar individualmente até $100 \mathrm{~N} / \mathrm{cm}^{2}$ de pressão, dispostos em uma área de $400 \times 400 \mathrm{~mm}$ de superfície ativa. O baropodômetro é composto de um conversor analógico digital de 16 bits e frequência de amostragem de $150 \mathrm{~Hz}$. Documenta as análises com imagens de pontos de pressão plantar e armazena todas as informações adquiridas a nível estático, bem como as disfunções funcionais do equilíbrio e estabilidade postural, sendo que as aquisições das imagens são precisas, instantâneas, repetíveis e não invasivas. A baro estática realiza a estabilometria e fornece o valor da superfície plantar em centímetros quadrados $\left(\mathrm{cm}^{2}\right)$ (BANKOFF et al., 2007). Em análises estabilométricas, as oscilações do centro de pressão são aleatórias e não estruturadas (DUARTE, 2000). A estabilometria consiste em avaliar as oscilações do corpo, em indivíduos mantidos em pé, sobre uma 
plataforma durante um tempo determinado (MARTINS, 2010). Desta forma, os sujeitos foram instruídos a permanecerem em apoio bipodálico, com os pés ligeiramente separados por uma largura confortável (de acordo com a largura dos ombros) e com os braços relaxados ao longo do corpo. O teste foi aplicado na posição estática, sendo dividido em quatro etapas: o primeiro com pés afastados e olhos abertos (PAOA), o segundo, com pés afastados e olhos fechados (PAOF), o terceiro de pés juntos e olhos abertos (PJOA), e por último, pés juntos e olhos fechados (PJOF). O teste foi executado uma veze para cada indivíduo, tendo duração de 30 segundos cada teste, aplicados um após o outro, sem intervalo. Para o reteste foi usado o mesmo padrão do teste.

Os dados foram apresentados por meio da estatística descritiva (média \pm desvio padrão). A normalidade dos dados foi testada por meio do teste de Shapiro-Wilk, sendo a correlação entre as variáveis, feita pelo teste de Correlação de Pearson. Um nível de significância de $\mathrm{p} \leq 0,05$ foi utilizado, por meio do pacote estatístico SPSS v. 18.0.

\section{RESULTADOS}

Os dados descritivos das variáveis dos testes de equilíbrio e capacidade funcional encontram-se na tabela 1 .

Tabela 1: Estatística descritiva (mínima, máxima, média e desvio padrão) das variáveis.

\begin{tabular}{l|r|r|r}
\hline \multicolumn{1}{c|}{ Variáveis $(\mathrm{n}=31)$} & Mínima & Máxima & Média \pm DP \\
\hline TUG (seg.) & 5,25 & 8,50 & $6,86 \pm 0,84$ \\
\hline CAD (seg.) & 9,05 & 16,50 & $11,56 \pm 1,73$ \\
\hline DEG (seg.) & 3,16 & 5,38 & $4,10 \pm 0,5$ \\
\hline EEB (pontos) & 45 & 56 & $51,3 \pm 3,08$ \\
\hline Baro - PAOA $\left(\mathrm{cm}^{2}\right)$ & 0,27 & 6,89 & $1,44 \pm 1,43$ \\
\hline Baro - PAOF $\left(\mathrm{cm}^{2}\right)$ & 0,18 & 6,37 & $1,70 \pm 1,39$ \\
\hline Baro - PJOA $\left(\mathrm{cm}^{2}\right)$ & 0,70 & 13,47 & $3,85 \pm 2,79$ \\
\hline Baro - PJOF $\left(\mathrm{cm}^{2}\right)$ & 1,13 & 22,40 & $5,27 \pm 4,28$ \\
\hline
\end{tabular}

$P A O A=$ Pés afastados $e$ olhos abertos; PAOF = Pés afastados e olhos fechados; PJOA = Pés juntos e olhos abertos; PJOF = Pés juntos e olhos fechados; TUG = Timed $U p$ and Go; $C A D=$ sentar e levantar em uma cadeira; $D E G=$ subir degraus; $E E B=$ Escala de equilíbrio de Berg.

Na tabela 2 encontram-se os valores de correlação entre os testes de equilíbrio e capacidade funcional, onde somente foi encontrada uma alta e significativa correlação entre a EEB com os testes TUG $(r=-0,710 ; p=0,01)$, sendo que as demais variáveis não apresentaram correlação significativa. 
Tabela 2: Correlação entre os testes de equilíbrio e capacidade funcional.

\begin{tabular}{l|c|c|c|c|c}
\hline & EEB & Baro PAOA & Baro PAOF & Baro PJOA & Baro PJOF \\
\hline TUG & $-0,710^{*}$ & 0,170 & $-0,273$ & 0,063 & $-0,171$ \\
\hline CAD & $-0,017$ & $-0,241$ & $-0,283$ & $-0,353$ & $-0,003$ \\
\hline DEG & $-0,320$ & 0,001 & $-0,102$ & 0,074 & $-0,017$ \\
\hline
\end{tabular}

$P A O A=$ Pés afastados e olhos abertos; PAOF = Pés afastados e olhos fechados; PJOA = Pés juntos e olhos abertos; PJOF = Pés juntos e olhos fechados; TUG = Timed Up and Go; $C A D=$ sentar e levantar em uma cadeira; $D E G$ = subir degraus; $E E B=$ Escala de equilíbrio de Berg; correlação significativa * $p=0,01$.

\section{DISCUSSÃO}

O presente estudo teve como finalidade verificar se há associação entre equilíbrio e capacidade funcional em mulheres idosas. Na associação entre testes de equilíbrio com testes de capacidade funcional houve apenas correlação entre BERG e TUG. Os resultados entre EEB e TUG corrobora com o estudo de Gonçalves et al. (2009) que realizaram os testes de EEB e TUG em idosas com e sem histórico de quedas, onde as idosas sem histórico de quedas obtiveram melhor desempenho em ambos os testes. Podemos entender que histórico de quedas pode estar associado a diminuição da capacidade funcional. O mesmo estudo mostrou que sujeitos que realizaram o teste de Berg com maior pontuação também realizaram o teste de TUG em um tempo menor, apontando para o fato de que quanto melhor a capacidade de manutenção do equilíbrio corporal, melhor o desempenho em tarefas funcionais. Silva et al. (2008) também encontraram uma correlação negativa, ou seja, quanto maior a pontuação no EEB, menor o tempo de realização no teste de TUG. O estudo de Rodini et al. (2008) ao correlacionar o teste de EEB com o TUG em idosos saudáveis, que se assemelha a amostra do presente estudo, por se tratarem de idosas saudáveis, também encontrando correlação entre os testes, cita ainda o fato de serem testes de fácil aplicabilidade sem necessidade de treinamento, o que pode ter colaborado com os resultados encontrados.

O teste de CAD não apresentou correlação com os testes de equilíbrio. Para Camara et al. (2008) o ato de sentar e levantar pode estar relacionado à força muscular e não ao equilíbrio, o que pode explicar os resultados presentes. Silva et al. (2010) também trazem informações onde a capacidade física em idosos ativos e sedentários no teste de sentar e levantar de uma cadeira estão relacionados à força de membros inferiores. Além disso, importante observar que o teste de CAD é um teste dinâmico onde envolve outro tipo de equilíbrio, classificado como equilibro recuperado, que é a capacidade de retomar o equilíbrio em uma posição qualquer. Já o Baropodômetro avalia o equilíbrio estático, o que diferencia do ato de sentar e levantar de uma cadeira (TUBINO, 1984). 
O teste de DEG não teve correlação com nenhum dos testes de equilíbrio. O estudo de Marrara et al. (2012) sugere que em testes de subir degraus, os resultados estão relacionados a um melhor condicionamento físico. Trata-se de testes onde se tem um acréscimo do componente deslocamento vertical, contra a ação da gravidade, o que podemos entender como uma necessidade maior de força. Assim como no teste de $\mathrm{CAD}$, relacionar o $\mathrm{DEG}$ ao fator força e não ao equilíbrio. Mello et al. (2013) corroboram tal aspecto ressaltando a importância da força de membros inferiores em teste de subir degraus.

A utilização da baropodometria na análise do equilíbrio corporal é uma tecnologia recente e de poucos estudos (MARTINS, 2010). Para Bankoff (2004) vários fatores são importantes para o equilíbrio corporal, entre eles a prática de atividade física. Em nosso estudo, esta técnica não denotou relação com a capacidade funcional das idosas. Possivelmente, o fato de o teste avaliar o equilíbrio estático difira da maioria dos testes de capacidade funcional que apresentam característica dinâmica. O equilíbrio estático difere do equilíbrio dinâmico por se tratar da manutenção de uma postura particular do corpo com o mínimo de oscilação, enquanto no equilíbrio dinâmico a manutenção da postura, no decorrer do desempenho de uma habilidade motora, desordena a orientação do corpo (FIGUEIREDO et al., 2007; ECKERT et al., 1993; BANKOFF et al., 2004).

Ainda, o fato das idosas serem saudáveis e na grande maioria ativas $(74,2 \%)$ o equilíbrio não esteja comprometido. Um estilo de vida ativa, quando consideradas as atividades domésticas, esportiva e de lazer pode contribuir positivamente na manutenção da autonomia (AMORIM et al., 2002). O estudo e Araújo et al. (2010) ao analisar o efeito do exercício resistido sobre o equilíbrio em idosos saudáveis, cita que a subjetividade do termo "idosos saudáveis" pode sugerir a existência de resultados falsos positivos. Talvez um estudo com idosos não saudáveis possa trazer resultados diferentes.

\section{CONCLUSÃO}

O fato da amostra deste estudo ser constituída unicamente por idosas saudáveis, e na grande maioria fisicamente ativas, pode justificar parte de nossos resultados, sendo sugeridos novos estudos com um perfil de idosos mais acometidos pela perda do equilíbrio. Além disso, a distinção entre os tipos de equilíbrio empregados nos testes funcionais e na baropodometria pode justificar a ausência de correlações entre os testes. Assim, conclui-se com o presente estudo que somente o teste TUG apresentou uma alta e significativa correlação com o teste de EEB, indicando que o equilíbrio dinâmico tem importante função na capacidade funcional neste padrão de teste. Sendo que os demais testes funcionais, bem como a barapodometria não apresentaram correlação. 


\section{REFERÊNCIAS}

AMORIM, Paulo R. S. et al. estilo de vida ativo ou sedentário: impacto sobre a capacidade funcional. Revista Brasileira Ciências do Esporte, Campinas, v. 23, n. 3, p. 49-63, maio 2002.

ARAUJO, M. L. M. et al.; Efeitos dos exercícios resistidos sobre o equilíbrio e a funcionalidade de idosos saudáveis: artigo de atualização. Fisioterapia e Pesquisa, São Paulo, v.17, n.3, p.277-83, jul/set. 2010.

BANKOFF, Antonia D. P. et al; Estudo do equilíbrio corporal postural através do sistema de baropodometria eletrônica. Revista Conexões, v. 2, n. 2, p. 87-104, 2004.

BANKOFF, Antonia D. P. et al. Postura e equilíbrio corporal: um estudo das relações existentes. Revista movimento e percepção, v. 7, n. 10, p. 89 - 104, 2007.

BERG, Katherine O. et al. Measuring balance in the elderly: preliminary development of an instrument. Physiotherapy Canada, v. 41, n. 6, p. 304-311, 1989.

BOUCHARD, Claudete. Atividade Física e Obesidade. Tamboré, SP: Cia Editorial, 2003.

BRASIL. Ministério da Saúde: envelhecimento e saúde da pessoa idosa [online]; 2007; Brasília. Cadernos de atenção básica. Brasília: 2007

CAMARA, Fabiano M. et al. Capacidade funcional do idoso: formas de avaliação e tendências. ACTA FISIATR, v. 15, n. 4, p. 249-256.

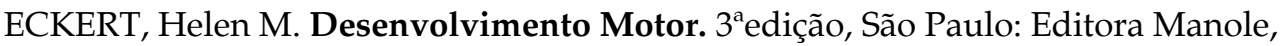
1993.

FIGUEIREDO, Karina M. O. B.; LIMA, Kênio C.; GUERRA, Ricardo O. Instrumentos de avaliação do equilíbrio corporal em idosos. Revista Brasileira de Cineantropometria e Desempenho Humano, v. 9, n. 4, p. 408-413, 2007.

GONÇALVES, Daniele F. F. et. al. Equilíbrio funcional de idosos da comunidade: comparação em relação ao histórico de quedas. Revista Brasileira de Fisioterapia, v. 13, n. 4, p. 316-23, 2009.

DUARTE, Marcos. Análise estabilográfica da postura ereta humana quase estática. Dissertação (Livre Docência em Educação Física) - Escola de Educação Física e Esporte, Universidade de São Paulo, São Paulo, 2000.

Instituto Brasileiro de Geografia e Estática - IBGE. Tábua completa de mortalidade para o Brasil. 2016.

IPAQ (2005). Guidelines for Data Processing and Analysis of the Internacional Physical Activity Questionnaire. Disponível em: <http:/www.ipaq.ki.se/>. Acesso em 05 junho. 2017. 
MARRARA, Kamilla T. et al. Responsividade do teste do degrau de seis minutos a um programa de treinamento físico em pacientes com DPOC." Jornal brasileiro de pneumologia, v. 38, n.5, 2012.

MARTINS, Maria S. E. Eficiência da estabilometria e baropodometria estática na avaliação do equilíbrio em pacientes vestibulopatas. Dissertação (mestrado). Universidade de Brasília, 2010.

MELLO, Paula M. S. et al. Tradução, adaptação cultural e validação de uma escala para aferir limitação da atividade de subir e descer escadas. Revista brasileira de geriatria e gerontologia, v. 16, n. 3, 2013.

NETO, João P. et al. Mobilidade funcional em função da força muscular em mulheres idosas fisicamente ativas. Revista Brasileira de Medicina do Esporte, v. 21, n. 5, 2015.

PATERSON, Donald H.; WARBURTON, Darren E. R. Physical activity and functional limitations in older adults: a systematic review related to Canada's Physical Activity Guidelines. International Journal of Behavioral Nutrition and Physycal Activity, v. 7, n. 38, 2010.

REBELATTO, Calvo J. R. et al. Influência de um Programa de Atividade Física de Longa Duração Sobre Força Muscular Manual e a flexibilidade Corporal de Mulheres Idosas. Revista Brasileira de Fisioterapia, v. 10, n. 1, p. 127-132.

RIBEIRO, Fernando. et al. Impacto da prática regular de exercício físico no equilíbrio, mobilidade funcional e risco de queda em idosos institucionalizados. Revista Portuguesa de Ciências do Desporto, v. 9, n. 1, p. 36-42, 2009.

RODINI, Carolina. et al. Estudo comparativo entre a Escala de Equilíbrio de Berg, o Teste Timed Up \& Go e o Índice de Marcha Dinâmico quando aplicadas em idosos hígidos. ACTA FISIATR 2008; v.15, n.4: 267 - 268.

RODRIGUES, Crystyano F. Avaliação da capacidade funcional e do equilíbrio corporal postural em idosos com e sem histórico de quedas. Dissertação (Mestrado em biologia oral) - Universidade do sagrado coração, Bauru - SP, 2015.

SHUMWAY-COOK, Anne et al. Predicting the Probability for Falls in CommunityDwelling Older Adults Using the Timed Up \& Go Test. Journal of the American Physical Therapy Association, v. 80, n. 9, p. 896-903, 2000.

SILVA, Andressa et al. Equilíbrio, Coordenação e Agilidade de Idosos Submetidos à Prática de Exercícios Físicos Resistidos. Revista Brasileira de Medicina do Esporte, v. 14, n. 2, 2008.

SILVA, Andressa F. et. al. Comparação da capacidade funcional e força de preensão palmar entre idosos caidores e não caidores. 2014. Trabalho de Conclusão de Curso (Fisioterapia), Belo horizonte, 2014. 
SILVA, Tamara O. et al. Avaliação da capacidade física e quedas em idosos ativos e sedentários da comunidade. Revista Brasileira de Clínica Médica, v. 8, n. 5, p. 392398.

SOUZA, Raquel F. et. al. Correlation between the body balance and functional capacity from elderly with chronic vestibular disorders. Brazilian Journal of Otorhinolaryngology, v. 77, n.6, p. 791-798.

TIGGEMANN, Carlos L. et al. Effect of traditional resistance and power training using rated perceived exertion for enhancement of muscle strength, power, and functional performance. The Official Journal of the American Aging Association, v. 38, n. 42, p. 1-12, 2016.

TUBINO, Manoel J. G. Metodologia científica do treinamento desportivo. $3^{\mathrm{a}}$ edição. São Paulo: Ibrasa, 1984. 